\title{
Bauern in theatralen Aktionsräumen: verlachenswert, vorbildlich, revolutionär
}

\author{
HeIdy GReCO-KaufMANN (BERN)
}

Der Titel „Bauern in theatralen Aktionsräumen“ bezieht sich einerseits auf Bauern als Figuren auf der Marktplatzbühne und im Rahmen des fastnächtlichen Brauchtums, dargestellt durch Angehörige der Luzerner Oberschicht, und anderseits auf Bauern als Protagonisten von theatralen Handlungen anlässlich des Konflikts zwischen Stadtherren und ländlichen Untertanen, der zum Bauernkrieg von 1653 führte. Anhand der unterschiedlichen theatralen Ereignisse wird die obrigkeitliche Inszenierung der Bauernfiguren in Fastnachtspiel und Brauchtum und die Selbststilisierung der Bauern im Rückgriff auf den Schweizer Befreiungsmythos aufgezeigt.

Bauernfiguren sind die wichtigsten dramatis personae der im 15. und 16. Jahrhundert zur Zeit des Karnevals aufgeführten Fastnachtspiele. Fastnachtspiel und „Bauernspiel“ galten beinahe als Synonyme. Als die komischen Bühnenfiguren par excellence belustigen die Bauern das Publikum durch derb-sinnliche Späße und drastische Gestik. Besonders in der sattsam bekannten frühen Nürnberger Tradition hat sich eine typisierte Darstellungsweise herausgebildet, die den Bauern die Rolle als grotesk-komische Vertreter der Vitalsphäre zuweist, deren Reden und Agieren sich meist um Sexuelles, Fäkales und Handgreiflichkeiten dreht.

Im Unterschied zu den meisten Nürnberger Fastnachtspielen sind die Bauern der Schweizer Spiele aber nicht durchwegs stereotyp als ungeschlachte dörper oder Bauern-Narren gezeichnet, sondern variieren in ihrer Gestaltung je nach der Funktion, die ihnen von den Fastnachtspiel-Autoren, die in der Regel der Machthabenden Schicht angehörten, zugedacht war. Im Extremfall kann gar eine radikale Umwertung der Bauernfigur vom triebhaft-dumpfen Tölpel zum frummen edlen pur, der sich durch Scharfsinn und Selbstbewusstsein auszeichnet, beobachtet werden. ${ }^{1}$ Die differenziertere und auf die jeweils intendierte Wirkung abgestimmte Charakterisierung der Bauernfigur in den Schweizer Spielen hängt mit den anders gelagerten politischen Verhältnissen, der unterschiedlichen Trägerschaft der Aufführungen - Patrizier statt Handwerker - und der späteren Blütezeit dieser Spielgattung in der Eidgenossenschaft zusammen. Die starke Verwurzelung schweizerischer Fastnachtspiele im Zeit-

1. Schmidlin, Stephan. Frumm byderb lüt. Ästhetische Form und politische Perspektive im Schweizer Schauspiel der Reformationszeit. Bern/Frankfurt/New York: Lang, 1983; Weishaupt, Matthias. Bauern, Hirten und ,frume edle puren". Bauern und Bauernstaatsideologie in der spätmittelalterlichen Eidgenossenschaft und der nationalen Geschichtsschreibung der Schweiz. Basel/ Frankfurt: Lang, 1992. 
geschehen hat dazu geführt, dass sie von der Forschung gerne als "Sonderentwicklungen “ abgegrenzt und als "politische Spiele“ etikettiert werden. ${ }^{2}$

Zuerst werde ich am Beispiel von Luzerner Fastnachtspielen aus dem 16. Jahrhundert versuchen, das spezifisch politisch gefärbte Bild, das sich in den Bauernfiguren verdichtet, zu skizzieren. Vorauszuschicken ist allerdings, dass die Luzerner Bühnenfiguren zwar von ihrer Aufmachung her bäuerisch-derb in Erscheinung treten, dass sie jedoch nicht ausschließlich Vertreter des Bauernstandes darstellen, sondern gleichermaßen den gemeinen Mann, d. h. den Untertan, verkörpern. An die Untertanen, die auch eigens zu den Aufführungen eingeladen wurden, richteten sich die Spiele hauptsächlich, denn der Weinmarkt, der im ökonomischen und politischen Zentrum der Stadt Luzern situierte Aufführungsort, war für das Luzerner Patriziat ein erstklassiges Forum, um Konflikte aller Art darzustellen und Botschaften zu vermitteln, wobei Fragen des Zusammenlebens und Alltagsprobleme im Spiel ebenso zur Darstellung gelangten wie handfeste politische Meinungsverschiedenheiten und Auseinandersetzungen, die das Gemeinwesen und die bestehenden Machtverhältnisse bedrohten. ${ }^{3}$ Im 1546 aufgeführten „Marcolfus“ thematisierte Zacharias Bletz anhand der altbekannten und als Volksbuch weit verbreiteten Fabel um den pfiffigen Bauern Markolf und den weisen König Salomon den sich verschärfenden Konflikt zwischen der städtischen Obrigkeit und den ländlichen Untertanen, der durch die rasche Entwicklung und Machtausdehnung der Stadt und die gleichzeitig wachsende Verschuldung der Landschaft entstanden ist.

Getrieben von der Sorge um das tägliche Brot fordert der Bauer Markolf den reichen und gelehrten König Salomon zu einem komischen Wettstreit heraus und erhofft sich so, eine existenzsichernde Stelle an seinem Hof zu ergattern. Amüsiert durch die grobschlächtige Erscheinung und die bauernschlaue Eloquenz seines Untergebenen lässt sich Salomon auf ein rhetorisches Kräftemessen ein, muss jedoch sehr bald feststellen, dass er mit dem gewitzten Bauern nicht zu Rande kommt. Für die Dauer der Aufführung herrscht nun die „verkehrte Welt“, der Niedere mokiert sich über den Höheren, der Weise muss vom Törichten lernen, die Sinneswelt triumphiert über die Geisteswelt. Dabei ist hinzuzufügen, dass der Luzerner Markolf nicht nur mit Salomon im Clinch ist, sondern ebenso mit den Höflingen, die ihn verspotten und gegen den Willen des Herrschers drangsalieren. Doch der Bauer beweist sowohl in Worten wie auch in Taten seine Überlegenheit.

2 Catholy, Eckehard. Fastnachtspiel, Stuttgart: Metzler, 1966. S. 76; Brett-Evans, David. Von Hrotsvith bis Folz und Gengenbach. Eine Geschichte des mittelalterlichen deutschen Dramas. Bd. 2. Berlin: Schmidt, 1975. S. 174; Frey, Winfried/Raitz, Walter/Seitz, Peter. Einführung in die deutsche Literatur des 12. bis 16. Jahrbundert. Bd. 2. Opladen: Westdeutscher Verlag, 1982. S. 120.

3 Für Einzelheiten verweise ich auf meine Publikationen: Greco-Kaufmann, Heidy. Vor rechten lütten ist guot schimpfen. Der Luzerner Marcolfus und das Schweizer Fastnachtspiel des 16. Jahrbunderts. Bern: Lang, 1994; dies. Spiegel dess vberflusses und missbruchs. Renward Cysats "Convivii Process". Kommentierte Erstausgabe der Tragicocomedivon 1593. Zürich: Chronos, 2001. 
Markolf ist für die kleinen Leute eine ideale Identifikationsfigur: Wie viele von ihnen hat er unter bedrängten wirtschaftlichen Verhältnissen zu leiden und träumt davon, seine Situation zu verbessern; stellvertretend für alle Unterprivilegierten demonstriert er Selbstbewusstsein und Scharfsinn und nimmt es mit den Mächtigen auf. Aus dem Munde des Fastnachtspiel-Helden können die Zuschauer, die sonst keine politischen Rechte haben, einmal im Jahr, an der Fastnacht, tüchtig ihre - sonst nicht gefragte - Meinung sagen. Im Spiel werden die normalen Verbote und Tabus aufgehoben, die soziale Ordnung vorübergehend außer Kraft gesetzt, den vitalen Trieben wird ihr Recht zugestanden, Aggressionen dürfen ausgelebt werden. Am Schluss des Fastnachtspiels wird allerdings unmissverständlich klar gemacht, dass die Zeit des Rollentausches, der Umkehrung der Verhältnisse, vorbei ist und die alte Ordnung wieder ihre Gültigkeit hat: Salomon ist wieder König und Markolf wieder Knecht. Dennoch hat Markolf erreicht, was er wollte: Er hat eine Anstellung bei Hofe erhalten und seine existenziellen Probleme gelöst. Während Markolf und Salomon in trauter Eintracht den alt-neuen sozialen Bund besiegeln, stehen die Höflinge, die Bediensteten des Herrschers, als die großen Verlierer da; deren Einflussbereich wird rigoros beschnitten. Die Marcolfus-Handlung, die zeitweilig den Charakter eines Protests gegen die herrschende soziale Ordnung hat, ist in Wirklichkeit dazu ausersehen, diese zu erhalten und zu stabilisieren. In den Schlussversen preist Markolf Salomon als gerechten König, der auch Spaß versteht, die Hofleute hingegen denunziert er als die eigentlichen Bauernfeinde, vor deren Boshaftigkeit nur gewarnt werden kann: Vor rechten lütten ist guot schimpfen. Aber vor den luren ist nüt zuo glimpfen. Hät ich denen gfolget, ich bät müssen hangen, Und wär mir dsel am strick usgangen. ${ }^{4}$

Die im Spiel vorgeführte Konstellation, König und Hofleute versus Bauernvertreter, lässt sich unschwer auf die damaligen Luzerner Machtverhältnisse übertragen: Eine aus wenigen Familien bestehende patrizische Oberschicht lenkte in oligarchischer Herrschaft die Geschicke des städtischen und landschaftlichen Territoriums, sekundiert durch eine mit Privilegien ausgestattete aufstrebende Bürgerschaft, der man aber keine Mitsprache einräumen wollte. Die ländlichen Gebiete waren von den Stadtherren abhängig, konnten ihre Position aber im Zuge der konfessionellen Auseinandersetzungen zeitweise stärken.

Durch das Vorführen des cleveren bäuerlichen Helden versuchten die „Herren von Gottes Gnaden“ der Landbevölkerung zu schmeicheln und deren Loyalität zu sichern, wobei der auf Konsens ausgerichtete Schluss ein friedvolles Nebeneinander gemäß der alten Ständeordnung propagiert. Den Höflingen, die Bletz so konsequent negativ gezeichnet hat, kommt dabei die Sündenbockrolle zu; die gegen die Obrig-

4 Steiner, Emil (Hg.). Die dramatischen Werke des Luzerners Zacharias Bletz. Frauenfeld/Leipzig: Huber, 1926. hier: „Marcolfus“, Vers 1798 f. Übersetzung: „Mit (ge-)rechten Leuten lässt sich gut scherzen, hinterlistigen Menschen aber kann man nichts recht machen. Hätte ich denen gehorcht, hätte ich hängen und mein Leben am Strick aushauchen müssen.“ 
keit aufgestauten Aggressionen verlagern sich auf sie. Ein kluger Schachzug, die Patrizier waren nämlich an mehreren Fronten engagiert: Zum einen bedurften sie der Bauern, um den neuen Glauben aufzuhalten und zu bekämpfen, mussten diese also umwerben damit sie nicht zum Feind überliefen, zum anderen wollten sie ihre absolutistischen Machtansprüche durchsetzen, mussten also die Bauern von der Notwendigkeit der Unterwerfung und des Gehorsams überzeugen. Zudem galt es, einer allfälligen Allianz von Bauern und Emporkömmlingen der städtischen Bürgerschaft, die nach Teilhabe an der Macht strebten, vorzubeugen.

Die Bauernfigur, die in der Luzerner Version des Marcolfus vom rebellischen zum vorbildlichen Untertan mutierte, wird in einem anderen, ebenfalls von Zacharias Bletz verfassten, 1567 aufgeführten Fastnachtspiel vom Wunderdoktor als verlachenswertes Opfer eines Wanderarztes und dessen Komplizen, des Wurzkrämers, gestaltet. ${ }^{5}$ Der Bauer Hans Latz wird angelockt durch das Geschrei des Krämers, der seine und die Dienste des Marktplatzheilers anpreist:

Wurm samen gGtt wurtzen zen vs brechen

Dryax vnd ougen fäll $z G$ stechen

Das han ich gedryben manig mall

Vnd kann das vs der masen woll

Klaper defeli han ich ouch feyll

Woll her woll her geb üch heyll

Wer nit zG stGll mag gan der kum

Den gib ich pillulen mumyum

Der Bauerntölpel Hans Latz sucht Hilfe für seine kranke Mutter, die an Bauchgrimmen und unaufhörlichem Durchfall leidet. In allen Details beschreibt er dem Krämer die Auswirkungen des Leidens, das die alte Bäuerin plagt. Gegen stattliches Entgelt händigt ihr der Kräuterhändler eine obskure Mixtur aus und erteilt auch gleich Ratschläge zur Anwendung:

Sä da bastu ein gGtte purgatz

(Abführmittel)

Die bring der $m$ Gtter und beb sy starck

Das ir nit glich empfall das marg...

(Mark)

Zufrieden trottet der Bauer mit dem vermeintlichen Wundermittel davon und macht den anderen Patienten Platz, die sich ebenfalls vom Wurzkrämer und vom landfahrenden Doktor behandeln lassen wollen. Die übrigen Ratsuchenden, wie der einfältige Hans Latz einfache Menschen aus dem Volk, wenden sich ebenso vertrauensvoll an die Marktplatzmediziner und schildern ihre Sorgen, die meist mit dem

5. Das noch unveröffentlichte Fastnachtspiel „Der Wunderdoktor“von Zacharias Bletz wird in der Zentral- und Hochschulbibliothek Luzern unter der Signatur Ms. 183 fol. aufbewahrt. Textauszüge bei Brandstetter, Renward. „Ueber Luzerner Fastnachtspiele“ Zeitschrift für dentsche Pbilologie 17 (1885). S. 421-431. (Eine Edition ist geplant). 
Geschlechtsleben und der Verdauung zusammenhängen, in drastischen Worten. Das Geschäft mit den vom Wunderdoktor verschriebenen und vom Wurzkrämer bereitgestellten Aphrodisiaka, Potenzmitteln und Pülverchen gegen das Rumoren in den Gedärmen läuft ausgezeichnet und beschert dem medizinischen Zweiergespann einen prallvollen Geldbeutel, den sie triumphierend vor den Augen des Publikums herumschwingen.

Wie im Wunderdoktor werden auch im anonymen, vermutlich aus der Feder von Zacharias Bletz oder Hans Salat stammenden Faßnacht spil von Astrology vnd warsagren, das nach einem ähnlichen Schema aufgebaut ist, dem Publikum die Dummheiten und Betrügereien von selbsternannten Doktoren, Astrologen und Wahrsagern vorgeführt. ${ }^{6}$ Gleich zu Beginn dieses Spiels disputieren zwei Bauern mit zwei Narren über die Tüchtigkeit des Doctor Rossschwantz, der angeblich alle Krankheiten heilen und zudem mit Hilfe seiner astrologischen Kenntnisse die Zukunft voraussagen kann. Je ein Bauer und ein Narr verteidigen die Gelehrtheit des Sterndeuters, während die beiden anderen dessen Künste mit derben Worten entlarven und ins Lächerliche ziehen. Der schlaue Bauer versucht vergeblich den Gutgläubigen von der Absurdität der Rossschwantzschen Praktiken zu überzeugen und resümiert:

Lieber, schweig, du machst mich zlachen

Bey goll, ich glaub, du seyst nitt bachen.

Während im Wunderdoktor die unlauteren Machenschaften des Quacksalbers und seines Gehilfen im Sinne einer negativen Didaxe unkommentiert zur Schau gestellt werden, kommt im Faßnacht spil von Astrology und warsagren ein dialektisches Verfahren zur Anwendung, das die Meinungen der Anhänger und Gegner kontrastiert. Die intendierte Wirkung ist jedoch dieselbe: Das Vorführen der gerissenen Betrüger und ihrer unbedarften Opfer in grotesk-komischen Szenen soll bei den Zuschauern Gelächter, Spott, Distanzierung und Überlegenheitsgefühle auslösen. Dass der aufklärerische Impetus dieser Fastnachtspiele tatsächlich Früchte trug und die Bauern und einfachen Leute zu einer kritischeren Einstellung gegenüber Marktplatzheilern bewegte, ist jedoch zu bezweifeln. Die von der Obrigkeit wiederholt erlassenen Mandate wie beispielsweise jenes aus dem Jahre 1590, das die Wegweisung aller vrömden scharlatanen vnd vmb strychenden banckschrygern, die sich für Artzet vssth Gend, aber die lütt verderbend ${ }^{7}$ befiehlt, lässt eher das Gegenteil vermuten.

Die nach einer schriftlichen Textvorlage in Szene gesetzten Fastnachtspiele betreffen jedoch nur den hochartifiziellen Spezialfall im Gros der fastnächtlichen Aktivitäten, die alljährlich die Stadt in einen Ausnahmezustand versetzten. Das im Volk so beliebte wilde goukelwäsen und das butzen- und böggenwerck, wie man das anarchische Getümmel und Maskentreiben damals nannte, wurde von der Luzerner

6 Das Spiel ist abgedruckt bei Bächtold, Jakob. „Quellen zu ,Aller Praktik Grossmutter““. Vierteljahresschrift für Literaturgeschichte. 3. (1890). S. 208-227.

7 Staatsarchiv Luzern, AKT 1, F4, Schachtel 773 B (3). 
Obrigkeit seit der zweiten Hälfte des 16. Jahrhunderts mit zunehmender Heftigkeit bekämpft, da sie Gewalttätigkeiten, Sittenzerfall und herrschaftsgefährdende Aktionen fürchtete. Weil mit Verboten wenig auszurichten war, wählte das Patriziat ein raffinierteres Vorgehen, um den unerwünschten Mummenschanz unter Kontrolle zu bringen. Das Aufführen von bis zu zwei Tage dauernden Fastnachtspielen, das aktive Fastnächtler zu passivem Zuschauen verführte, war eine mögliche Maßnahme, eine andere war das Unschädlichmachen von Brauchtumsgestalten durch gezielte Modifizierung und Integration in herrschaftskonforme Schauhandlungen. Quellenmäßig am besten dokumentiert ist dieses Vorgehen bei der Fritschifigur, über die chronikalische Einträge bis ins 15 . Jahrhundert zurückreichen und die - nebenbei bemerkt - bis heute als zentrale Figur der Luzerner Fastnacht nichts von ihrer Faszination verloren hat. Die ersten Aufzeichnungen über die Anfänge des Fastnachtsbrauchs liefert uns Diebold Schilling (geboren um 1460, gestorben vermutlich 1515), Verfasser der Luzerner Bilderchronik von 1513.

Von alter bar ist ein lobliche gewonheit und $j$ (rlicher faßnacht schimpf [ Scherz] $z G$ Lucern gew(sen, uff eine gesellschaft und trinckstuben, genant zum Fritschi. Die hand einen str=winen man, genannt brGder Fritschi, den sy j(rlich uff den schmutzigen donstag vor der pfaffenvasnacht erlich in irem harnesch mit allen gesellschafften der statt Lucern mit eim vennli, pfiffen, trummen, tantzen und was sich mag $z G$ fr $=$ uden zieben, infFrend. ${ }^{8}$

Irritierend ist zunächst einmal die Bezeichnung ströwin man. Nach Paul Hugger handelt es sich bei diesem um eine Brauchtumsfigur, die bezüglich Sinngehalt und Funktion dem im ganzen Alpengebiet anzutreffenden Wilden Mann vergleichbar ist. ${ }^{9}$ Dass in Luzern Stroh statt Tannzweige als Verkleidungsmaterial verwendet wurde, leuchtet ein, denn Luzern war damals ein wichtiger Umschlagplatz des nahen Kornanbaugebietes. Hugger vermutet denn auch, dass Schilling mit besagtem ströwinen man eine ältere, vorzünftische Maskengestalt bezeichnete, die an der Fastnacht des einfachen Volkes ihr Unwesen getrieben habe und die der Chronist Schilling nicht mehr aus eigener Anschauung kannte. Interessant ist nämlich, dass der in der Bilderchronik abgebildete Fritschi offensichtlich eine Holzmaske darstellt, die nicht so recht zu einem Strohgewand passt. Auch die nachfolgende Schilderung, welche die Vorgänge rund um den in fastnächtlichem Geist inszenierten Raub des Bruder Fritschi durch die Basler wiedergibt, bezeugt, dass sich der einstmals wilde Strohgesell längst zu einer „anständigen“ Brauchtumsgestalt gemausert hatte, die man am Schmutzigen Donnerstag im Beisein der Standesorganisationen mit militärischen Ehren in die Stadt begleiten konnte. Noch deutlicher wird die Verein-

8 Schmid, Alfred A. (Hg.). Die Schweizer Bildercbronik des Luzerners Diebold Schilling, 1513. Luzern: Faksimile-Verlag, 1981. S. 393.

9 Hugger, Paul. „Bruder Fritschi von Luzern. Zur Deutung einer fastnächtlichen Integrationsfigur". Schweizerisches Archiv für Volkskunde 79 (1983). S. 113-128. 
nahmung dieser anfänglich grob-bäuerischen Maskengestalt durch die gesellschaftliche Elite, wenn man die von Stadtschreiber Renward Cysat (er lebte von 1545 bis 1614) gegen Ende des 16. Jahrhunderts verfasste Ursprungslegende beizieht, in der die Fritschifigur auf eine angeblich historische Persönlichkeit mit Namen Fridolin an der Halden zurückgeführt wird. Cysat erzählt, dass der im Volksmund Bruoder Fritschi Genannte ein guoter landtman vnd vßburger diser statt ... vnd ouch ein kriegsman gewesen und um 1480 gestorben sei. ${ }^{10}$ Er habe sich das Jahr hindurch pflichtbewusst synes anerbornen puwrenbandels ond-wäsens angenommen und sei nur zur Fastnacht in die Stube der Safranzunft gekommen, um in guter Gesellschaft zu zechen. In Erinnerung an diesen vorbildlichen Bauern und ehemaligen Söldner führe nun Rat und Bürgerschaft jeweils am Schmutzigen Donnerstag einen Umzug durch, wobei die wehrfähigen Männer, nach Waffengattungen gruppiert, den in einem weißblauen Rock, den Luzerner Stadtfarben, einher reitenden Fritschi begleiteten.

Während die Schauhandlungen rund um den zum Patrioten und vorbildlichen Bauern verklärten Bruoder Fritschi von der Obrigkeit noch ausgebaut wurden, mehrten sich gleichzeitig die Fastnachtsverbote: Larven-und Butzenwerck wurde nicht mehr geduldet.

Dieser fragmentarische Einblick in das „offizielle“, vom Luzerner Patriziat getragene Spiel- und Brauchtumswesen mag genügen, um zu belegen, dass die verschiedenen theatralen Handlungen weitgehend der Absicht entsprangen, obrigkeitliche Politik auf populäre Weise dem Volk schmackhaft zu machen. Mit ihren Aufführungen zielen die Machthaber gleichermaßen auf die Lenkung, Belehrung und Persuasion der Zuschauer. Ob im Rahmen von literarischen Fastnachtspielaufführungen oder brauchtümlichen Schauhandlungen, letztlich agieren alle Bauernfiguren im Sinne des Patriziats und bieten als Spaßmacher Ersatz für die gefürchteten fastnächtlichen Bräuche, entlarven gesellschaftsschädigende Praktiken und sittlich-moralische Vergehen und verkünden politische Botschaften.

Obrigkeitlich instrumentalisierte Schauereignisse sind zahlreich und gut dokumentiert, theatrale Aktivitäten von Untertanen hingegen äußerst selten nachweisbar und meist nur im Zusammenhang mit Konflikten aktenkundig. Beim folgenden Beispiel, in dem es um theatrale Aktivitäten seitens der Untertanen geht, schlüpften die Bauern, vielleicht dem Vorbild der Herren folgend, ebenfalls in Kostüme und inszenierten bühnenreife Aktionen, um ihre Anliegen zu vertreten. Schauplatz war das Luzerner Hinterland, zu den Zuschauern und schließlich unfreiwilligen Akteuren gehörten Vertreter des Luzerner Rates. Situiert sind die Ereignisse im Umfeld der Auseinandersetzungen des sogenannten Bauernkriegs von 1653. ${ }^{11}$ Auslöser war

10 Cysat, Renward. Collectanea Chronica und denkwürdige Sachen pro Cbronica Lucernensi et Helvetis. Hg. Josef Schmid. Erster Band, zweiter Teil. Luzern: Diebold Schilling Verlag, 1969. S. 718 .

11. Die relevanten Fakten entnehme ich den akribischen Forschungen des Historikers Andreas Suter, der in seiner Habilitationsschrift eine Gesamtschau der Ereignisse rund um den Bauern- 
eine durch benachbarte Territorialherren ausgelöste Geldentwertung, welche die Entlebucher besonders hart traf, weil ihnen im Unterschied zu anderen Untertanen die Währungsverluste nicht ersetzt wurden. Am 9.1.1653 wandte sich in dieser Angelegenheit eine Delegation der Talschaft an den Rat der Stadt Luzern. Weil sich die abwertenden Orte weigerten, Kompensationen an fremde Untertanen auszurichten, wies auch die Luzerner Obrigkeit die Bitte zurück. Die abschlägige Antwort wurde von den Bauern mit Erbitterung aufgenommen; überliefert ist die Reaktion von Poley Christen aus Hasle, der die herrschaftliche Absage folgendermaßen kommentiert haben soll: „daß min Gnädige Herren nur den Tellen mit ihnen spielen". ${ }^{12}$ Mit dieser Deutung der Situation stellte der Hasler Bauer den Bezug zum schweizerischen Befreiungsmythos her und beschwor die kollektive Erinnerung an die Figur Wilhelm Tell und seine Miteidgenossen, die in heroischem Kampf die tyrannischen Vögte vertrieben oder gar getötet hatten. Die Parallelisierung der damaligen mit den aktuellen Verhältnissen musste den erbosten Entlebuchern, die schon lange unter den Verletzungen legitimer Ansprüche und Beschneidungen ihrer Rechte zu leiden hatten, wie eine Offenbarung vorgekommen sein, denn fortan spielte das alte Freiheitssymbol eine herausragende Rolle im Konflikt zwischen den "gnädigen Herren“ und ihren bedrängten Untertanen. Die Bauern rotteten sich zusammen, veranstalteten Prozessionen und illegale Landsgemeinden und verbündeten sich gegen die Luzerner Obrigkeit. Zu den Drahtziehern der Rebellion gehörten Hans Zemp und Caspar Undernärer aus Schüpfheim sowie Ueli Dahinden aus Hasle, die sich anlässlich eines Bauerntreffens am 10. Februar 1653 nicht nur verbal mit der Symbolgestalt Tell auseinander setzten, sondern ihrer Identifikation mit dem unbeugsamen Freiheitshelden durch einen öffentlichen Auftritt in historischen Kostümen, finanziert vom Entlebucher Pannermeister Hans Emmenegger, Ausdruck verliehen. Undernärer „habe ein armbrust und köcher getragen und Wilhelmen Tell repräsentieren“ sollen, der neben ihm schreitende Dahinden habe „einen sack und drei stein darinnen“ getragen und „den Stauffacher von Schwytzen fürbilden sollen“, der dritte im Bunde war Hans Zemp „und er habe ein ax getragen und Erne im Melchtal bedüten" sollen. ${ }^{13}$ In dieser Aufmachung nahmen die drei Aufwiegler auch an einem Umzug teil, der vor die Herberge führte, in der die Delegation des Luzerner Rates logierte, die nach Schüpfheim gekommen war, um die unbotmäßigen Untertanen zu maßregeln. Die „drei Tellen“, wie die als alte Eidgenossen verkleideten Männer auch genannt wurden, bildeten zusammen mit Alphornbläsern und „Knüttelhauptleuten“ die Vorhut des Zuges von insgesamt 1400 mit Prügeln bewaffneten

krieg bietet: Suter, Andreas. Banernkrieg von 1653. Tübingen: Bibliotheca-Academica-Verlag, 1997.

12 Staatsarchiv Luzern: 13/3570, 12. Januar 1653, zit. in: Suter, Andreas. Bauernkrieg von 1653. Tübingen: Bibliotheca-Academica-Verlag, 1997, S. 92.

13 Staatsarchiv Zürich: A 223.1 zit. in: Suter, Andreas. Banernkrieg von 1653. Tübingen: Bibliotheca-Academica-Verlag, 1997. S, 144. 
Männern, die an den herrschaftlichen Gesandten vorbeidefilierten. Die Vertreter des Luzerner Rats waren erschrocken und empört über das selbstbewusste Auftreten der Bauern und lehnten deren Forderungen ab mit dem Hinweis, dass Verhandlungen nur in der Stadt geführt werden dürften. In ihrer „Klag über unsere Unterthanen des Land Entlebuochs" beschwerten sie sich insbesondere über deren unziemliche Umgangsformen: „Si haben auch gegen uns den gewonlichen stylum wie underthanen gebuert verenderet. " ${ }^{14}$ Die Stadt Luzern begegnete der Revolte mit harter Repression, die Anführer wurden für vogelfrei erklärt und unerbittlich gejagt. Undernärer und Dahinden, zwei der Tellen, flüchteten vor den anrückenden Truppen der Luzerner in die Entlebucher Alpen, Zemp, der dritte Tell, setzte sich ins Elsass ab. Die Landbevölkerung unterstützte die Flüchtenden und gewährte ihnen Unterschlupf und Nahrung. Die Tellen, an deren Seite nun der ebenfalls verfolgte Hans Stadelmann, der einem Tagsatzungsboten den Bart geschoren hatte, als Ersatz für Zemp auftrat, waren zu Identifikationsfiguren geworden. Das Dreiergespann nahm weiterhin an konspirativen Bauerntreffen teil, an denen der bäuerliche Widerstand koordiniert wurde. So auch am 28. September 1653, als in Schüpfheim eine Gruppe wütender Aufständischer die Ereignisse des Tages diskutierte.

Was war geschehen? Am traditionellen Schwörtag, zu dem die Territorialherren ihre Untertanen eingeladen hatte, um die herrschenden Machtverhältnisse zu bestätigen, verkündete ein Bauernvertreter, dass er nur bereit sei, Gehorsam zu schwören, wenn die Gefangenen und für vogelfrei Erklärten begnadigt würden. Der Landvogt lehnte dieses Begehren schroff ab; ein weiterer Affront gegen die Aufständischen, der das Fass zum Überlaufen brachte. Noch in derselben Nacht beschlossen die in die Enge getriebenen Drei Tellen, ihre selber gewählte Rolle zu Ende zu spielen und dem historischen Vorbild folgend den Tyrannenmord zu vollbringen. Zum Zeichen der Verbundenheit mit dem Helden aus der Gründerzeit der Eidgenossenschaft trugen sie ein letztes Mal ihre Kostüme, die roten und schwarzen Schwyzer Kleider mit den weißen Kreuzen auf der Brust ${ }^{15}$ und legten sich am folgenden Tag beim Hohlweg „Buggenschachen" in den Hinterhalt, um den verhassten Herren auf ihrem Heimritt nach Luzern aufzulauern. Damit stellten sie exakt jene aus der Befreiungssage bekannte Szene nach, nur überschritt die nachfolgende Tat das „als ob“ einer theatralen Handlung, „der Geist der Dargestellten schien sich auf die Darstellenden übertragen zu haben "16 und so erschossen sie den Luzerner Zeugherrn Caspar Studer und verwundeten den Schultheiss Dulliker am Bein, wobei der erfolgreiche Schütze Undernärer triumphierend verkündete, er

14 Staatsarchiv Luzern: 13/3585 zit. in: Suter, Andreas. Banernkrieg von 1653. Tübingen: Bibliotheca-Academica-Verlag, 1997. S. 145.

15 Vgl. Chronik Katzengrau, zit. in: Suter, Andreas. Bauernkrieg von 1653. Tübingen: BibliothecaAcademica-Verlag, 1997. S. 301.

16 Marchi, Otto: Schweizer Geschichte für Ketzer oder Die wundersame Entstehung der Eidgenossenschaft. Zürich: Praeger, 1971. S. 73. 
habe „den dellen schuss" getan. ${ }^{17}$ Die Tat wurde von der ländlichen Bevölkerung offenbar als Akt ausgleichender Gerechtigkeit empfunden, denn die drei Tellen wurden noch am selben Abend ins Schüpfheimer Rathaus eingeladen und konnten am nächsten Tag unbehelligt die Messe besuchen und öffentlich ihre Tatwaffen zur Schau stellen. Damit vollbrachten sie einen weiteren symbolischen Akt, mit dem sie sich der Kirchengemeinschaft als Werkzeuge Gottes empfahlen. Die Unterstützung weiter Teile der Bevölkerung blieb den drei Attentätern auch dann noch erhalten, als die Luzerner Obrigkeit mit äußerster Brutalität durchgriff, Truppen in Schüpfheim stationierte, für deren Unterhalt die Untertanen aufzukommen hatten, und Angehörige in Sippenhaft nahm. Schließlich führte jedoch der Verrat eines Unbekannten, der den Lockungen des auf die Tyrannenmörder ausgesetzten Kopfgeldes nicht widerstehen konnte, zum Aufspüren des Verstecks in der Nähe von Schüpfheim. Nach dem Bericht eines Chronisten wurden Undernärer und Dahinden nach verzweifelter Gegenwehr von den Soldaten ,wie Vögel“ vom Scheunendach geschossen. Der dritte Tell, Hans Stadelmann, konnte zunächst fliehen, wurde aber später ebenfalls verraten, und zwar vom inzwischen aus dem Elsass zurückgekehrten Hans Zemp, der ursprünglich ebenfalls als einer der drei Tellen in Erscheinung getreten war. Der letzte Akt des Dramas wurde in Luzern gespielt, die Regie lag nun wieder fest in den Händen der „gnädigen Herren“. Stadelmann wurde am 15. Juli 1654 enthauptet, sein Kopf auf den Haberturm gespießt und sein Leib auf dem Hochgericht von Luzern ans Rad geflochten. Die Leichen der im vergangenen Herbst erschossenen Attentäter wurden nachträglich nach Luzern überführt und am 9. Oktober ebenfalls enthauptet, gevierteilt und auf dasselbe Rad geflochten. Mit diesem öffentlich inszenierten "Theater des Schreckens ${ }^{\text {"18 }}$ wurde die grenzenlose, sozusagen über den Tod hinaus reichende Macht der Herrschenden und die Ohnmacht der Bauern demonstriert.

Die durch Spieltexte überlieferten Aufführungen auf dem Weinmarkt, die fastnächtlichen Schauhandlungen mit dem gezähmten Bruder Fritschi, die symbolträchtigen theatralen Aktivitäten aufmüpfiger Bauern und die drakonischen Straf- und Abschreckungsrituale der Machthaber zeichnen sich bei aller Disparatheit durch eine signifikante Gemeinsamkeiten aus: Alle Formen der Schauereignisse wurden von gesellschaftlichen Gruppen bewusst in Szene gesetzt, um politische Ziele zu erreichen. Die Herrschenden bedienten sich bei ihrer theatral inszenierten Machtpolitik so lange der komischen Bauernfigur, bis ihnen die bäuerlichen Untertanen unmissverständlich klarmachten, dass sie nicht länger gewillt waren, die Rolle der unbedarften und leicht beeinflussbaren Narren zu spielen. Der entschlossenen Selbstdarstellung der Bauern im Gewande des Freiheitshelden, der dreisten Usurpation der nationalen Symbolgestalt, konnten die Herren nicht mehr mit Komik, sondern nur noch mit tödlichem Ernst begegnen.

17 Staatsarchiv Luzern: 13/3825 (Verhörakten Stadelmann) zit. in: Suter, Andreas. Banernkrieg von 1653. Tübingen: Bibliotheca-Academica-Verlag, 1997. S. 301.

$18 \mathrm{Zu}$ diesem Thema vgl. auch die Untersuchungen von van Dülmen, Richard. Theater des Schreckens. Gerichtspraxis und Strafrituale der Früben Neuzeit. München: Beck, 1985. 